\title{
Adverse Outcomes of Underuse of B-Blockers in Elderly Survivors of Acute Myocardial Infarction
}

\section{Citation}

Soumerai, Stephen B. 1997. "Adverse Outcomes of Underuse of ?-Blockers in Elderly Survivors of Acute Myocardial Infarction." JAMA: The Journal of the American Medical Association 277 (2) (January 8): 115. doi:10.1001/jama.1997.03540260029031.

\section{Published Version}

doi:10.1001/jama.1997.03540260029031

\section{Permanent link}

http://nrs.harvard.edu/urn-3:HUL.InstRepos:32696164

\section{Terms of Use}

This article was downloaded from Harvard University's DASH repository, and is made available under the terms and conditions applicable to Other Posted Material, as set forth at http:// nrs.harvard.edu/urn-3:HUL.InstRepos:dash.current.terms-of-use\#LAA

\section{Share Your Story}

The Harvard community has made this article openly available.

Please share how this access benefits you. Submit a story.

\section{Accessibility}




\title{
Adverse Outcomes of Underuse of $\beta$-Blockers in Elderly Survivors of Acute Myocardial Infarction
}

\author{
Stephen B. Soumerai, ScD; Thomas J. McLaughlin, ScD; Donna Spiegelman, ScD; \\ Ellen Hertzmark, MA; George Thibault, MD; Lee Goldman, MD
}

Objectives.-To study determinants and adverse outcomes (mortality and rehospitalization) of $\beta$-blocker underuse in elderly patients with myocardial infarction; and whether the relative risks (RRs) of survival associated with $\beta$-blocker use were comparable to those reported in the large randomized controlled trials (RCTs).

Setting.-New Jersey Medicare population.

Design.-Retrospective cohort design using linked Medicare and drug claims data from 1987 to 1992.

Patients. - Statewide cohort of 5332 elderly 30 -day acute myocardial infarction (AMI) survivors with prescription drug coverage, of whom 3737 were eligible for $\beta$-blockers.

Main Outcome Measures.- $\beta$-Blocker and calcium channel blocker use in the first 90 days after discharge and mortality rates and cardiac hospital readmissions over the 2-year period after discharge, controlling for sociodemographic and baseline risk variables.

Results.-Only $21 \%$ of eligible patients received $\beta$-blocker therapy; this rate remained unchanged from 1987 to 1991. Patients were almost 3 times more likely to receive a new prescription for a calcium channel blocker than for a new $\beta$-blocker after their AMls. Advanced age and calcium channel blocker use predicted underuse of $\beta$-blockers. Controlling for other predictors of survival, the mortality rate among $\beta$-blocker recipients was $43 \%$ less than that for nonrecipients (RR=0.57; $95 \%$ confidence interval $[\mathrm{Cl}], 0.47-0.69)$. Effects on mortality were substantial in all age strata (65-74 years, $75-84$ years, and $\geq 85$ years) and consistent with the results for elderly subgroups of 2 large RCTs. $\beta$-Blocker recipients were rehospitalized $22 \%$ less often than nonrecipients (RR=0.78; $95 \% \mathrm{Cl}, 0.67-0.90)$. Use of a calcium channel blocker instead of a $\beta$-blocker was associated with a doubled risk of death (RR=1.98;95\% Cl, 1.44-2.72), not because calcium channel blockers had a demonstrable adverse effect, but because they were substitutes for $\beta$-blockers.

Conclusions. $-\beta$-Blockers are underused in elderly AMI survivors, leading to measurable adverse outcomes. These data suggest that the survival benefits of $\beta$-blockade after an AMI may extend to eligible patients older than 75 years, a group that has been excluded from RCTs.

JAMA. 1997;277:115-121 and the Department of Medicine, University of California, San Francisco (Dr Goldman).

An earlier version of this article was presented at the 1996 annual meeting of the Society for General Internal Medicine, Washington, DC, May 4, 1996

Reprints: Stephen B. Soumerai, ScD, Department of Ambulatory Care and Prevention, Harvard Medica School and Harvard Pilgrim Health Care, 126 Brookline Ave, Suite 200, Boston, MA 02215
B-BLOCKER prophylaxis after acute myocardial infarction (AMI) is one of the most scientifically substantiated, cost-effective preventive medical services. ${ }^{1}$ Multiple randomized controlled trials (RCTs), involving over 20000 patients, have shown that $\beta$-blocker use following AMI decreases cardiovascular mortality and reinfaretions and increases the chances of survival by $20 \%$ to $40 \%{ }^{2-6}$ This evidence has led national cardiology consensus committees to strongly recommend their use in eligible populations of AMI patients. ${ }^{7}$ However,

For editorial comment see p 155.

few data exist on rates and determinants of prescription of $\beta$-blockers, especially in community settings and among elderly patients who have been underrepresented in RCTs. ${ }^{8}$ Although $80 \%$ of all deaths due to AMI occur in the elderly, virtually no patients older than 75 years have been included in RCTs of $\beta$-blockers. ${ }^{8-10}$ In this cohort study, we linked several large administrative databases on survival and use of inpatient and outpatient health care services to measure levels, determinants, and outcomes of prescribing $\beta$-blockers among a community population of 5332 elderly 30-day survivors of AMI in New Jersey.

Although claims databases can identify variations in practice patterns $\mathrm{s}^{11,12}$ and evaluate quality-of-care effects of costcontainment policies, ${ }^{13,14}$ the validity of their use in assessing the outcomes of adherence to evidence-based practice recommendations is unknown. ${ }^{15-19}$ To clarify the potential utility of claims-based outcomes research, trialists have recom- 
mended that the results of such observational studies should be compared with the results of RCTs of 6 technologies whose effects are unambiguous, including the effects of $\beta$-blockade on survival after an $\mathrm{AMI} .^{20}$

In this study, we sought to answer the following specific questions: (1) What proportion of eligible elderly AMI patients receive $\beta$-blocker prophylaxis after AMI? (2) Controlling for differences in risk status, are patient characteristics (age, sex, race, socioeconomic status [SES]) and use of alternative medications (eg, calcium channel blockers) associated with receipt of $\beta$-blockers in eligible patients? (3) Is the nonuse of $\beta$-blockers among eligible patients associated with increased mortality and rehospitalization for cardiovascular illness following AMI, controlling for potentially confounding patient variables? (4) Are the relative mortality rates associated with $\beta$-blocker use obtained in this observational study comparable to those reported for elderly subgroups of large RCTs?

\section{METHODS}

\section{Data Sources}

We linked 3 large, longitudinal databases: (1) New Jersey Medicare hospital admissions (part A) and enrollment data for a $100 \%$ sample of AMI patients from 1986 to 1992; (2) New Jersey Medicaid drug utilization and enrollment files for a $100 \%$ sample of Medicaid patients for the years 1986 to 1991; and (3) the New Jersey Program of Pharmacy Assistance for the Aged and Disabled (PAAD) drug utilization data for nonMedicaid elderly for a $100 \%$ sample of enrollees from 1986 to 1991.

Using the Part A Medicare files, we determined the admission and discharge dates, primary and secondary diagnoses, and procedures associated with the first (index) AMI hospitalization (which occurred during 1986-1990) and with all other hospital admissions in the year prior and 2 years following the index AMI. Medicare enrollment (HISKEW [Health Insurance Skeletonized Writeoff file]) data also included demographic characteristics (age, sex, race) as well as a record of survival and date of death up to 2 years following the index AMI. The reliability of these data for ascertaining mortality is well established. ${ }^{21}$

Medicaid and PAAD drug claims data contained complete and reliable longitudinal histories of the dates and identities of outpatient drug prescriptions, ${ }^{14,22,23}$ which were provided at no charge (or for a small co-payment) to New Jersey Medicare beneficiaries enrolled in Medicaid or PAAD. Individual
PAAD enrollees were ineligible for Medicaid but nonetheless had incomes of less than $\$ 19000$ in 1991.

\section{Cohort Definition}

We constructed a sample of Medicare patients with AMI enrolled in Medicaid or PAAD between 1986 and 1990, using inclusion and exclusion criteria almost identical to those in previous studies of the outcomes of AMI among Medicare populations. $^{24}$

Inclusion Criteria.-We identified elderly persons (aged 65 years and older) discharged from a hospital from 1986 to 1990 with a principal diagnosis of AMI (International Classification of Diseases, Ninth Revision [ICD-9] codes 410.0410.9). A recent study comparing such principal diagnoses with independently derived diagnoses obtained from hospital charts indicated high sensitivity (94\%) and predictive value $(92 \%){ }^{25}$ We increased predictive value further using the exclusions described below. We defined the index AMI admission for each patient as the first AMI admission after January 1, 1987, but before January 1, 1991 (AMIs in 1986 were used only to identify previous AMIs for the 1987 cohort).

Exclusion Criteria.-We excluded the following: (1) patients with end-stage renal disease or those residing outside New Jersey; (2) those hospitalized with an AMI in the 12 months preceding their index hospitalization; (3) patients who died during the incident admission or within 30 days of discharge (to ensure a minimum time window for measuring outpatient use of $\beta$-blockers); (4) those not enrolled in either drug benefit program for at least 6 months before the index admission and at least 30 days after discharge; and (5) patients discharged alive whose length of stay for the index AMI admission was less than 5 days, indicating a possible miscoding of AMI diagnosis. ${ }^{26}$

\section{Contraindications to $\beta-B l o c k e r$ Use}

We defined our primary study group as AMI patients who met the inclusion and exclusion criteria described above, and had no measurable absolute or relative contraindications to prophylactic use of $\beta$-blockers. ${ }^{7}$ Although it was impossible to identify all patients who had specific contraindications to $\beta$-blockade, we identified diagnoses and medications used before the index admission that represented potential contraindications to $\beta$-blocker use (for example, furosemide as an indicator of severe congestive heart failure [CHF], which was considered an absolute contraindication to $\beta$-blocker use at the time). Therefore, we eliminated from analysis all persons with 1 or more prescriptions for furosemide in the 6 months before the index admission. We also considered a principal hospital diagnosis of heart failure (ICD-9 code 428) in the prior year as a marker for severe $\mathrm{CHF}$ and an absolute contraindication to $\beta$-blockers; this measure has been found to have moderately high sensitivity $(85 \%)$ and positive predictive value ( $87 \%$ ) in hospital claims data. ${ }^{25}$ Since angiotensin-converting enzyme (ACE) inhibitors and digoxin are also used for heart failure we included them as covariates in the analyses (Table 1).

Since asthma is also considered a contraindication to $\beta$-blockers, ${ }^{7}$ patients who used oral or inhaled bronchodilators (eg, theophylline) in the 6 months before the index AMI, or who had a principal or secondary hospital discharge diagnosis of asthma or chronic obstructive pulmonary disease before their AMI were excluded from our analysis. Since insulindependent diabetes is a relative contraindication to $\beta$-blockers, we eliminated from analysis all patients with any prescriptions of insulin during the 6 months before the index AMI.

\section{Dependent Variables}

For the analyses of predictors of $\beta$-blocker use, the dependent variable was the time to first outpatient use of any $\beta$-blocker following the index AMI within the first 90 days after discharge. We also conducted sensitivity analyses using a time window of 1 month after discharge as a more specific definition of prophylactic $\beta$-blocker use. However, this definition did not change any of our findings. The reference time for the analysis was the day of discharge from the index admission; patients were followed until a $\beta$-blocker was dispensed or the patient was censored from the analysis either through death, loss of eligibility, or end of the predefined time window.

In the second phase of analysis, we studied the relationship of $\beta$-blocker exposure with 2 patient outcomes, adjusting for the clinically relevant and significant predictor variables described below. The primary outcome variable was mortality (time to death), because this is the most frequent measure used in RCTs of $\beta$-blockers and can be measured reliably. Randomized trials have documented that $\beta$-blockers reduce nonfatal reinfarctions by $25 \%$ to $30 \%$, and reduce morbidity from other cardiac conditions by similar amounts. ${ }^{82}$ Therefore, we also investigated the relationship between use of a $\beta$-blocker and time to new admission for any of the following conditions: AMI (ICD-9 code 410); angina (ICD-9 code 413); other ischemic heart disease (ICD-9 code 411), CHF (ICD-9 code 428), or other cardiovascular diseases (eg, essential hypertension) (ICD-9 codes 401, 402, 416, 424426 , and 785). 


\section{Independent Variables}

Patient variables, such as age, sex, race, and Medicaid enrollment, have all been associated with differing levels of access to drug therapies and cardiac technologies as well as survival ${ }^{27-29}$; therefore, all analyses of $\beta$-blocker use and outcomes adjusted for these variables (see Table 1). Because virtually all elderly Medicaid patients had yearly incomes below $\$ 6000$ compared with $\mathrm{PAAD}$ recipient incomes of up to $\$ 19000$ per year, this binary variable (Medicaid vs PAAD) also represented a reliable indicator of relative poverty or SES.

Indicators of severity of illness (Table 1) included the number of hospital admissions in the year prior to the index hospitalization with a principal diagnosis of a cardiac condition (angina, ischemic heart disease, CHF), presence of principal or secondary admission diagnoses for $\mathrm{CHF}$, angina, other ischemic conditions, and other cardiovascular diseases (eg, essential hypertension); use of drugs in the 6 months before the AMI as markers for specific cardiovascular conditions (digoxin, ACE inhibitors, and antianginal agents); and use of ACE inhibitors in the 90 days after the index AMI (marker of possible $\mathrm{CHF}$ ). We also included categorical variables for use of $\beta$-blockers and calcium channel blockers in the 6 months before the AMI, because they were likely to predict use of these agents after the index AMI, as well as several control variables measuring the length of stay of the index admission, and whether the patient underwent revascularization.

Potential indicators of comorbidities are also included in Table 1 . We measured the number of noncardiac hospitalizations (based on principal diagnoses) in the year before the index AMI, as well as the number of secondary diagnoses at the index admission. Also, 4 specific categories of secondary diagnoses (all cancers, chronic renal failure, cerebrovascular disease, and pneumonia) were included because they were significantly associated with a $40 \%$ or higher 2-year mortality rate in comparison with all Medicare patients. We also constructed an index indicating the number of different drug products taken in the 6 months before the AMI, because such variables have predicted adverse outcomes in our previous research. ${ }^{13}$

\section{Statistical Analysis}

We used Cox proportional hazards regression models to measure the effect and relative importance of patient characteristics on speed of access to $\beta$-blocker therapy following AMI. ${ }^{30}$ The dependent variable in these analyses was the timeto-patient use of $\beta$-blockers in the first 90 days after the index AMI. For all analy- ses, we included variables for age, sex, race, SES, and year of AMI regardless of their significance levels. All other risk adjustment and control variables were included in the final models only if they achieved a significance level of .10 or less in a stepwise regression procedure. Relative risk (RR) estimates contrasting the conditional probability of receiving $\beta$-blockers in 1 subgroup vs another (eg, 75-84 years old vs 65-74 years old), given that $\beta$-blockers had not already been received, were estimated by exponentiation of regression coefficients, and the $95 \%$ confidence intervals (CIs) were determined by standard methods..$^{30-32}$

The second phase of analyses estimated the effect of $\beta$-blocker use on survival times and time to new cardiac hospitalization, adjusting for the effect of any patient, severity, or comorbidity variables that independently predicted access to $\beta$-blockers and/or health outcomes, using Cox proportional hazards models as described above. As before, we included in the analysis all AMI study patients eligible for $\beta$-blocker treatment (ie, had no identifiable contraindications). We treated any use of $\beta$-blockers, ACE inhibitors, or calcium channel blockers within the first 90 days following the index admission as a time-varying covariate. Patients were censored due to death or disenrollment, but not for discontinuation of $\beta$-blocker therapy.

To ascertain the sensitivity of our primary results to residual confounding, we conducted 2 additional sets of analyses to adjust more completely for potentially confounding variables. In the first approach, which we termed the "saturated" model, we added to the list of candidate variables eligible for selection in stepwise regression all 2-way interactions of variables listed in Table 1 . In addition, we used $P=.30$ as the criterion for variable inclusion. This is a more conservative approach to control for confounding, and has been shown in simulations to perform well in this regard. ${ }^{33}$ In the second analysis, we used a propensity score approach. ${ }^{34}$ Propensity to exposure (in this case, $\beta$-blocker use by 90 days) scores were developed using saturated Cox regression models as described previously. Given all model covariate values, a propensity score was calculated for each study subject and was used to adjust the estimated effect of $\beta$-blocker use on mortality by entering this score as a single covariate in addition to $\beta$-blocker use in the models for these outcomes. In addition, subjects were stratified by tertiles of propensity for $\beta$-blocker use and stratum-specific estimates of the effect of $\beta$-blocker use on patient outcomes were obtained and compared, adjusted for propensity within strata.
Table 1.-Characteristics of Eligible Study Patients $(\mathrm{N}=3737)^{*}$

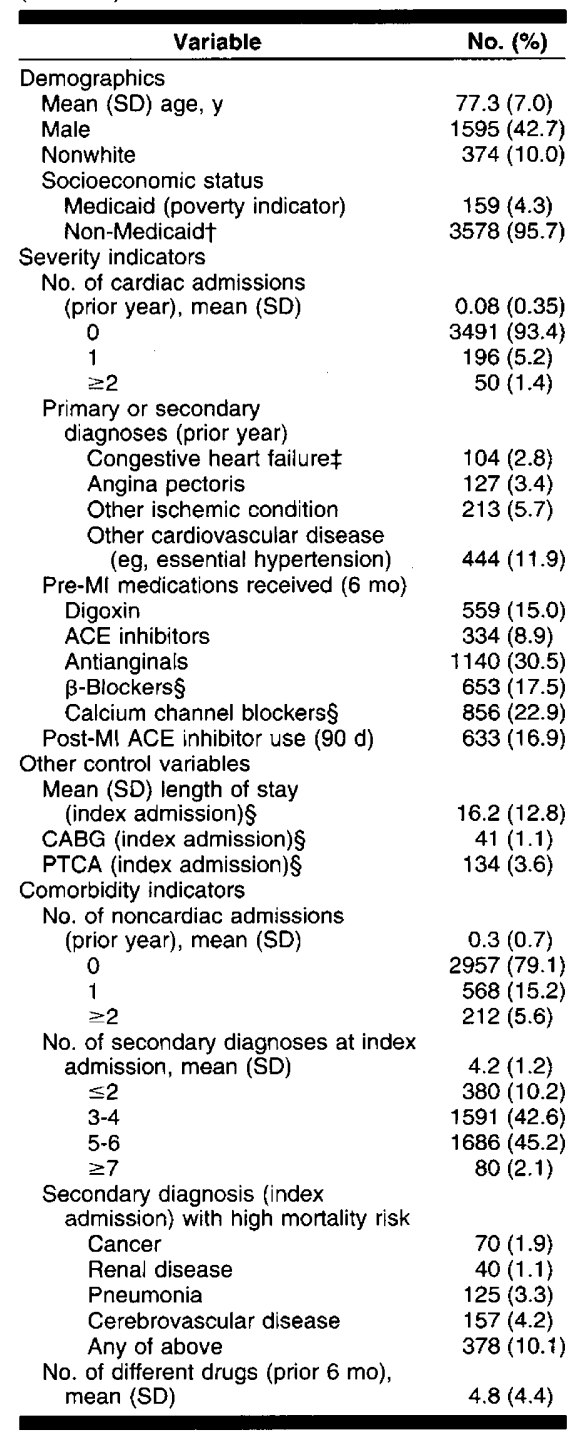

* Data expressed as No. (\%) unless otherwise specified. $\mathrm{Ml}$ indicates myocardial infarction; $\mathrm{ACE}$, angiotensin-converting enzyme; CABG, coronary artery bypass graft; and PTCA, percutaneous transluminal coronary angioplasty. †Moderately low- to middle-income individuals participating in program of pharmaceutical assistance for the elderly (New Jersey Program of Pharmacy Assistance for the Aged and Disabled maximum income, $\$ 19000$ per year [1991]).

tSecondary diagnosis only, because primary diagnosis of congestive heart failure resulted in ineligibility for study cohort.

$\S$ Control variables for both severity and practice style.

\section{RESULTS}

\section{Characteristics of Sample}

Of the 5332 elderly patients who met study criteria (see above), 1595 patients $(30 \%)$ had 1 or more absolute or relative contraindications to $\beta$-blocker treatment: $8.5 \%$ with an asthma admission or bronchodilator use, $9 \%$ with insulin use, and $19 \%$ with possible severe $\mathrm{CHF}$ (principal hospital discharge diagnosis of $\mathrm{CHF}$ or use of furosemide). This resulted in a final study sample of 3737 AMI patients who were defined as eligible for $\beta$-blocker therapy. 
Eligible study patients (Table 1) had a median age of 77 years, slightly more than one half of the study subjects were women, $10 \%$ were nonwhite, and $4 \%$ had very low incomes (Medicaid) compared with $96 \%$ (non-Medicaid) with low to moderate incomes.

One quarter of the study patients had been admitted to a hospital in the year prior to their index AMI admission, most often with a noncardiac diagnosis (Table 1). Twenty-three percent to $27 \%$ of the cohort members experienced their index AMI in each of the 4 years of accession into the study (1987-1990).

The high burden of illness of AMI in the elderly ${ }^{8,24}$ was reflected by a high rate of adverse events in the 2 years of followup. Of the cohort members, all of whom had survived 30 days, $17 \%$ died during the first year after their AMI; after 2 years of follow-up, $27 \%$ had died. A total of $33 \%$ experienced a new hospital admission with a principal diagnosis of AMI, angina, other ischemic heart disease, or $\mathrm{CHF}$ during the first year of follow-up.

\section{Outpatient Use of $\beta$-Blockers and Alternate Medications}

Only $21 \%$ of eligible study subjects received 1 or more prescriptions for a $\beta$-blocker in the 90 days following discharge from the index AMI admission. This rate of $\beta$-blocker use was essentially unchanged from the rate before the AMI (Table 1). The prevalences of $\beta$-blocker use after the AMI in the 1987, 1988,1989 , and 1990 cohorts were $20 \%$, $18 \%, 20 \%$, and $24 \%$, respectively.

Use of calcium channel blockers increased immediately after the AMI from $23 \%$ of patients in the 6 months before the index admission to $49 \%$ of AMI survivors during the first 90 days after hospital discharge. Among 2881 patients not receiving a calcium channel blocker before the AMI, $42 \%$ received 1 or more prescriptions from this drug class during the first 90 days after the AMI. In contrast, among 3084 patients not receiving a $\beta$-blocker before the AMI, only $15 \%$ were started on $\beta$-blocker therapy during the 3 months after the AMI. Similarly, $73 \%$ of recipients of calcium channel blockers before the AMI continued such therapy, compared with only $48 \%$ of recipients of $\beta$-blockers before the AMI who continued receiving $\beta$-blockade after discharge. The prevalences of use of calcium channel blockers in the 90 days after the AMI in the 1987, 1988, 1989 , and 1990 cohorts were $50 \%, 48 \%$, $43 \%$, and $54 \%$, respectively.

\section{Predictors of $\boldsymbol{\beta}$-Blocker Use}

Controlling for all other covariates in the proportional hazards model, patients aged 75 to 84 years and patients older

Table 2.-Independent Predictors of Trial of $\beta$-Blockers Within 90 Days of Discharge (Index Admission) Among Eligible Patients in a Proportional Hazards Regression Model*

\begin{tabular}{|c|c|c|}
\hline Variable & $\begin{array}{c}\text { Relative Risk of } \\
\text { B-Blocker Use, } \\
(95 \% \mathrm{Cl})\end{array}$ & $\boldsymbol{P}$ \\
\hline $\begin{array}{l}\text { Demographics } \\
\text { Age, y } \\
65-74 \dagger\end{array}$ & 1.0 & $\ldots$ \\
\hline $75-84$ & $0.86(0.74-0.99)$ & .04 \\
\hline$\geq 85$ & $0.56(0.44=0.73)$ & $<.001$ \\
\hline Male & $0.94(0.81-1.08)$ & .38 \\
\hline Nonwhite & $1.02(0.80-1.30)$ & .90 \\
\hline $\begin{array}{l}\text { Socioeconomic status } \\
\text { Medicaidt }\end{array}$ & 1.0 & $\ldots$ \\
\hline Non-Medicaid & $1.22(0.83-1.82)$ & .33 \\
\hline $\begin{array}{l}\text { Severity indicators } \\
\text { Hospital admission diagnosis (secondary) of } \mathrm{CHF} \text { (prior year) }\end{array}$ & $0.52(0.28-0.99)$ & .05 \\
\hline $\begin{array}{l}\text { Pre-Ml medications received }(6 \mathrm{mo}) \ddagger \\
\text { Digoxin }\end{array}$ & $0.71(0.56-0.90)$ & .004 \\
\hline Calcium channel blockers & $1.31(1.10-1.55)$ & .002 \\
\hline$\beta$-Blockers & $3.49(3.00-4.07)$ & $<.001$ \\
\hline Post-MI use of ACE inhibitors $(90 \mathrm{~d}) \ddagger$ & $0.58(0.40-0.85)$ & .005 \\
\hline $\begin{array}{l}\text { Comorbidity indicators } \\
\text { No. of hospital admissions (prior year)§ }\end{array}$ & $0.88(0.80-0.98)$ & .02 \\
\hline No. of secondary diagnoses at index admission§ & $0.92(0.87-0.98)$ & .005 \\
\hline Any one of high-risk secondary diagnoses\| & $0.79(0.60-1.04)$ & .09 \\
\hline No. of different drugs (prior $6 \mathrm{mo}$ ) $\S$ & $1.03(1.01-1.04)$ & .01 \\
\hline Post-MI use of calcium channel blockers $(90 \mathrm{~d}) \ddagger$ & $0.64(0.51-0.80)$ & $<.001$ \\
\hline
\end{tabular}

*Includes all sociodemographic variables, severity, comorbidity, and control variables from Table 1 with $P<.10$ in stepwise model. $\mathrm{Cl}$ indicates confidence interval; $\mathrm{CHF}$, congestive heart failure; MI, myocardial infarction; and $A C E$, angiotensin-converting enzyme.

theference category.

†Reference category: no use of each medication.

§Relative risk for each additional admission, diagnosis, or drug.

|Cancer, renal failure, pneumonia, or cerebrovascular disease.

than 85 years were $14 \%$ and $44 \%$, respectively, less likely than the "youngold" (ages 65-74 years) to receive a prescription for $\beta$-blockers $(\mathrm{RR}=0.86$ and 0.56 ; see Table 2). Sex, race, and SES were not independently associated with $\beta$-blocker treatment. The substantially higher use of calcium channel blockers shortly after the index infarction was associated with a $36 \%$ reduction in the likelihood of $\beta$-blocker use among eligible patients $(\mathrm{RR}=0.64 ; 95 \% \mathrm{CI}, 0.51$ 0.80 ).

\section{B-Blocker Use and Mortality}

Consistent with the RCT evidence, the receipt of a $\beta$-blocker among patients considered eligible for prophylactic $\beta$-blockade after the AMI was strongly and independently associated with a decreased mortality risk during 2 years of followup (Table 3). Controlling for all other predictors of mortality, the adjusted relative mortality rate among $\beta$-blocker recipients was about $43 \%$ less than for nonrecipients $(\mathrm{RR}=0.57 ; 95 \% \mathrm{CI}, 0.47$ $0.69)$.

The effect of $\beta$-blocker use on mortality rates was consistent and substantial in all age strata (Figure 1). The findings of this study were also consistent with the results of the 2 large RCTs that included substantial numbers of elderly patients (Figure 2).
The estimated RR of death among $\beta$-blocker recipients vs nonrecipients was unchanged when we used alternative methods to control more completely for possible confounding factors, such as adjustment by the propensity to use $\beta$-blockers obtained from the multivariate Cox regression models ( $R R=0.61 ; 95 \% \mathrm{CI}$, $0.50-0.74),{ }^{34}$ and by more highly saturated models that included all 2-way interaction terms with $P$ values less than or equal to .30. The adjusted relative mortality rate among $\beta$-blocker recipients vs nonrecipients was constant across tertiles of propensity to receive $\beta$-blockers and was also unaffected by exclusion of 623 additional patients with markers of CHF (any CHF diagnosis, use of digoxin with or without $\mathrm{ACE}$ inhibitors).

Using the estimated attributable mortality risk among those who did not receive $\beta$-blockers ( $43 \%$, see Table 3 ), approximately 381 of the 886 deaths occurring among these patients might have been avoided if they had been given $\beta$-blockers.

\section{Use of Calcium Channel Blockers and Mortality}

Controlling for all predictors of mortality listed in Table 3 , recipients of calcium channel blockers alone $(n=1380 \mathrm{pa}$ tients eligible for $\beta$-blockers) had nearly twice the risk of death as patients who 
Table 3.-Association of Receipt of $\beta$-Blocker Therapy With Mortality Over 2 Years of Follow-up, Controlling for Demographic, Severity, and Comorbidity Variables From Proportional Hazards Regression Model

\begin{tabular}{|c|c|c|}
\hline \multirow[b]{2}{*}{ Variable } & \multicolumn{2}{|c|}{ All-Cause Mortality } \\
\hline & $\begin{array}{c}\text { Relative Risk } \\
\text { (95\% Confidence Interval)* }\end{array}$ & $\boldsymbol{P}$ \\
\hline Receipt of $\beta$-blocker (first 90 d) & $0.57(0.47-0.69)$ & $<.001$ \\
\hline $\begin{array}{l}\text { Demographic variables } \\
\text { Age, y } \\
65-74 \dagger\end{array}$ & 1.0 & $\ldots$ \\
\hline $75-84$ & $1.46(1.26-1.70)$ & $<.001$ \\
\hline$\geq 85$ & $2.32(1.94-2.78)$ & $<.001$ \\
\hline Male & $1.34(1.18-1.52)$ & $<.001$ \\
\hline Nonwhite & $0.96(0.77-1.18)$ & .68 \\
\hline Non-Medicaid & $1.03(0.76-1.38)$ & .87 \\
\hline
\end{tabular}

*Controlling for all sociodemographic variables and the following severity, comorbidity, and control variables from Table 1 with $P<.10$ in stepwise model: pre-myocardial infarction (MI) use of: digoxin, angiotensin-converting enzyme (ACE) inhibitors, calcium channel blockers; post-MI use of ACE inhibitors; number of noncardiac admissions (prior year); number of secondary diagnoses at index admission; secondary diagnosis of cancer, renal failure, pneumonia, or cerebrovascular disease at index admission; number of different drugs (prior 6 months); coronary artery bypass graft during index admission; percutaneous transluminal coronary angioplasty during index admission; and length of stay of index admission.

†Reference category.
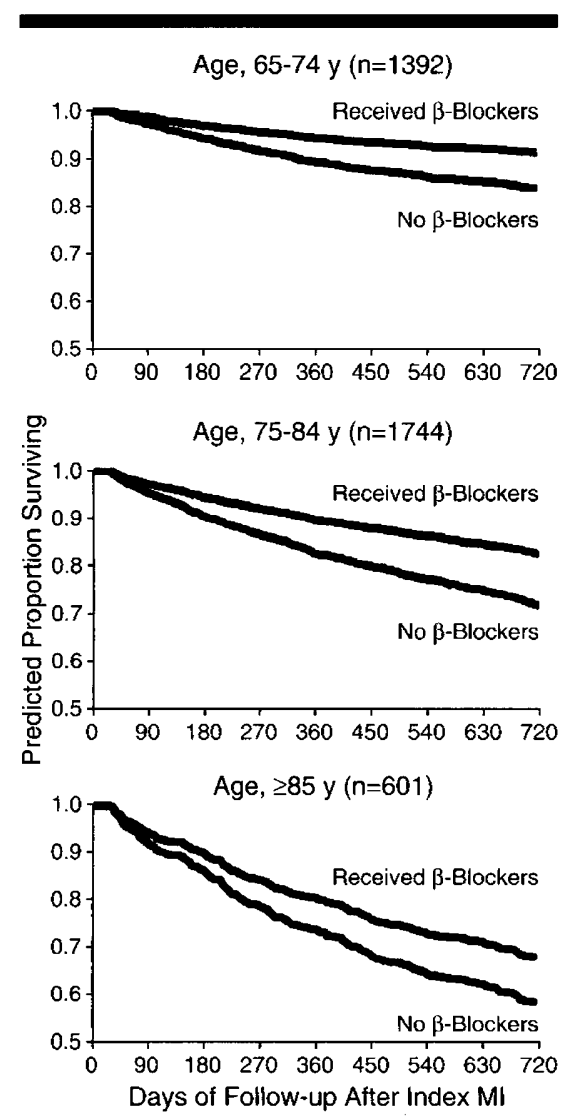

Figure 1.-Predicted survival for $\beta$-blocker recipients and nonrecipients stratified by age (age strata: $65-74$ years [top], 75-84 years [middle], $\geq 85$ years [bottom]). Kaplan-Meier curves, evaluated at the average value of all model covariates (Table 3 ) at baseline within each stratum, were used to construct this figure (adjusted relative risk $[\mathrm{RR}]=0.50$, $95 \%$ confidence interval [Cl], 0.36-0.72] for those aged $65-74$ years; $\mathrm{RR}=0.56,95 \% \mathrm{Cl}, 0.43-0.73$ for those aged $75-84$ years; and RR=0.72, $95 \% \mathrm{Cl}$, $0.47-1.11$ for those aged $\geq 85$ years).

received only $\beta$-blockers $\quad(n=334$; $\mathrm{RR}=1.98 ; 95 \% \mathrm{CI}, 1.44-2.72$ ). However, recipients of calcium channel blockers did not have a greater adjusted risk of indicating that the severity and comorbidity indicators used in our original model had adequately controlled for this type of confounding.

\section{B-Blocker Use and New Hospital Admissions}

Controlling for the number of cardiac admissions in the year before the index AMI and all other significant severity and comorbidity variables (Table 1), the risk of rehospitalization among $\beta$-blocker recipients was about $22 \%$ less than for nonrecipients $(\mathrm{RR}=0.78 ; 95 \% \mathrm{CI}, 0.67$ $0.90)$.

\section{COMMENT}

The findings of this study suggest that substantial opportunities exist for increased use of an inexpensive preventive therapy for reducing morbidity and mortality among elderly AMI patients. Advanced age, indicators of heart failure, and use of calcium channel blockers soon after the index AMI were strong indicators of nonreceipt of $\beta$-blocker therapy. Such underuse of $\beta$-blockers was consistently associated with increased mortality and rehospitalization, even among 2345 patients older than 75 years, an age group that has been consistently excluded from RCTs of longterm $\beta$-blockers after AMI.

Underuse of $\beta$-blockers among surviving AMI patients in the community is of growing concern to both specialists and generalists. ${ }^{1,2,4,35}$ Previous data on the prevalence of actual use of $\beta$-blockers in patients eligible for this therapy, while scant, are consistent with the findings of this study. ${ }^{9,10,36,37}$ Advanced age is a strong predictor of reduced use of $\beta$-blockers in the acute phase of illness, ${ }^{38,39}$ and was associated with lower use of these agents in outpatient settings in our study as well. Yet, paradoxically, the survival benefits of $\beta$-blocker therapy appear to be at least as great among older patients as compared with the nonelderly. ${ }^{8}$

Given their substantial beneficial effects, why is use of $\beta$-blockers so low? Based on a large 2-state survey, $50 \%$ of generalists and $75 \%$ of cardiologists believe that long-term $\beta$-blocker therapy "definitely improves survival." 35 Yet, lower actual $\beta$-blocker prescribing, as determined in this and previous studies, ${ }^{9,36,37}$ suggests that clinicians may know the "right" answer to survey questions regarding $\beta$-blocker therapy, but continue to omit such agents from patients' regimens. Barriers to $\beta$-blocker therapy may include mistaken beliefs that these agents are harmful or less beneficial for patients with left ventricular dysfunction or with diabetes (a relative contraindication), and exaggerated 
concerns regarding adverse effects on quality of life. ${ }^{1}$ For example, previous concerns regarding increased depression, fatigue, and reduced libido have proven to be unsubstantiated if $\beta$-selective agents are prescribed at the lowest effective doses. ${ }^{40,41}$ Extensive marketing of the newer calcium channel blockers may have also contributed to clinicians' negative attitudes toward $\beta$-blockers.

This study has several limitations. First, the study data did not allow us to identify heart block, sinus bradycardia, and severe peripheral vascular disease, which represent absolute or relative contraindications to $\beta$-blockade. Fortunately, however, we were able to analyze clinical data from a recently published medical record study in 37 Minnesota hospitals, ${ }^{38}$ which indicated that after excluding patients with insulindependent diabetes, asthma, or chronic obstructive pulmonary disease, or patients taking furosemide, only $6.7 \%$ of remaining elderly inpatients with AMI experienced any heart block, bradycardia, or severe peripheral vascular disease between the third day of admission and hospital discharge. Thus, assuming conservatively that $6.7 \%$ of the New Jersey cohort had these contraindications and did not receive $\beta$-blockers, the proportion of eligible patients receiving $\boldsymbol{\beta}$-blockers would have changed only marginally from $21 \%$ to $22.5 \%$.

A second limitation is our inability to measure obesity, smoking, or other lifestyle risk factors that are not contained in administrative data. However, this limitation would not affect our results unless such factors are associated with $\beta$-blocker use. Third, we could not identify patients who may have received $\beta$-blockers in the hospital, experienced an adverse drug reaction, and subsequently discontinued therapy before receiving an outpatient prescription. However, only a small fraction of elderly AMI patients receive oral $\beta$-blockers in hospitals ${ }^{42}$; only about $3 \%$ of patients are withdrawn from $\beta$-blockers due to an adverse drug reaction during the first 2 weeks after the $\mathrm{AMI}^{5}$; and patients with outpatient drug coverage are unlikely to receive more than a 1- or 2-day supply of medications at discharge.

Despite our attempts to control for confounding, it is possible that the higher risk associated with the use of calcium channel blockers reflects patients' higher intrinsic risks for $\mathrm{CHF}$ or other complications. In our cohort, patients receiving calcium channel blockers alone fared much worse than patients who received $\beta$-blockers alone. This higher mortality risk among calcium channel blocker recipients was very stable, even after multivariate adjustment for CHF and other risk factors; and this excess risk remained unchanged after excluding all patients with any markers of CHF. Our data add to the growing concern that calcium channel blockers should not be substituted for $\beta$-blockers in circumstances in which the latter have been proven to be effective. ${ }^{43}$ However, this study was not designed to answer questions regarding any independent adverse effects of calcium channel blockers, and $\beta$-blocker eligible patients who instead received calcium channel blockers had adjusted outcomes similar to patients who received neither.

The similarity of our estimates of the effects of $\beta$-blocker treatment on survival to estimates from several large RCTs provides evidence that cohort studies using administrative databases may sometimes be useful in estimating the outcomes of guideline adherence. A previous study using clinical records at 1 teaching hospital was also able to replicate the results of $1 \beta$-blocker RCT by carefully defining cohorts at risk so that they resembled the patient samples recruited for the RCTs. ${ }^{44}$ However, other studies of different drugs in surgery have observed uncontrollable selection biases that resulted in a greater likelihood for the retrospective comparisons to indicate treatment effectiveness when compared with the RCTs. ${ }^{19}$ We speculate that several characteristics of our population, technology, and data sets may have increased the likelihood of obtaining valid findings. First, patients experiencing a new AMI are a well-defined population, and our exclusion criteria also reduced the likelihood of misclassification. In addition, there are only a few contraindications to $\beta$-blocker treatment after AMI, and most of these could be measured in the database. Thus, a study of prophylactic use of $\beta$-blockers is less subject to confounding by indication than evaluation of other drug treatments that represent markers of serious illness and reduced survival (eg, ACE inhibitors following AMI). Finally, the relevant outcomes of $\beta$-blocker therapy (mortality and rehospitalization) could be measured reliably in Medicare data. However, additional research is needed on similar well-defined populations and other treatments with known effects before the validity and generalizability of such targeted outcomes research can be determined.

In summary, despite strong evidence demonstrating that use of $\beta$-blockers following AMI decreases morbidity and mortality, they are substantially underused in the elderly. Our findings suggest that this underuse leads to measurable adverse outcomes, including a
$43 \%$ excess risk of 2-year mortality and a $20 \%$ increase in rates of rehospitalization for cardiovascular disease. The apparent frequent substitution of calcium channel blockers for $\beta$-blockers following AMI is also associated with an increased mortality risk. This analysis of a large cohort of typical community patients provides strong support for existing guidelines to prescribe $\beta$-blockers instead of calcium channel blockers as a routine preventive therapy for elderly AMI patients.

This study was supported by grants RO1 HSO7631 and RO1 HSO6341 from the Agency for Health Care Policy and Research and by the Harvard Pilgrim Health Care Foundation.

We are indebted to Chris Pashos, PhD, and the Harvard Medical School Patient Outcomes Research Team for Acute Myocardial Infarction for assistance in Medicare data collection and cohort identification algorithms; to Mary Barton, MD, MPP, and Matthew Gillman, MD, SM, for their comments on an earlier version of the manuscript; to Jen Associates, Ine Cambridge, Mass, for linking the 3 databases and creating statistical analysis files; and to Ann Payson, MA, for technical assistance in the preparation of the manuscript and figures.

\section{References}

1. Kennedy HL, Rosenson RS. Physician use of beta-adrenergic blocking therapy: a changing per spective. I Am Coll Cardiol. 1995;26:547-552.

2. Goldman L, Sia STB, Cook EF, Rutherford JD, Weinstein MC. Costs and effectiveness of routine therapy with long-term beta-adrenergic antagonists after acute myocardial infarction. $N \mathrm{Engl} J \mathrm{Med}$ 1988;319:152-157.

3. Antman EM, Lau J, Kupelnick B, Mosteller F Chalmers TC. A comparison of results of metaanalyses of randomized control trials and recommendations of clinical experts: treatments for myocardial infarction. JAMA. 1992;268:240-248.

4. Lau J, Antman EM, Jimenez-Silva J, Kupelnick B, Mosteller F, Chalmers TC. Cumulative metaanalysis of therapeutic trials for myocardial infarction. N Engl J Med. 1992;327:248-254.

5. The Norwegian Multicenter Study. Timolol-induced reduction in mortality and reinfarction in patients surviving acute myocardial infarction N Engl J Med. 1981;304:801-807.

6. $\beta$-Blocker Heart Attack Trial Research Group. A randomized trial of propranolol in patients with acute myocardial infarction, I: mortality results. JAMA. 1982;247:1707-1714.

7. ACC/AHA Task Force. Guidelines for the early management of patients with acute myocardial infarction: a report of the American College of Cardiology/American Heart Association Task Force on Assessment of Diagnostic and Therapeutic Cardiovascular Procedures (Subcommittee to Develop Guidelines for the Early Management of Patients With Acute Myocardial Infarction). J Am Coll Car diol. 1990;16:249-292.

8. Jansen RWMM, Gurwitz JH. Controversies surrounding the use of $\beta$-blockers in older patients with cardiovascular disease. Drugs Aging. 1994;4: 175-183.

9. Eccles M, Bradshaw C. Use of secondary prophylaxis against myocardial infarction in the north of England. BMJ. 1991;302:91-92.

10. Brand DA, Newcomer LN, Freiburger A, Tian H. Cardiologists' practices compared with practice guidelines: use of beta-blockade after acute myocardial infarction. $J$ Am Coll Cardiol . 1995;26:14321436.

11. Chassin MR, Brook RH, Park RE, et al. Variations in the use of medical and surgical practices by the Medicare population. $N$ Engl J Med. 1986;314: 285-290. 
12. Wennberg JE. Dealing with medical practice variations: a proposal for action. Health Aff (Millwood). Summer 1984;3:6-32.

13. Soumerai SB, Ross-Degnan D, Avorn J, McLaughlin TJ, Choodnovskiy I. Effects of Medicaid drug-payment limits on admission to hospitals and nursing homes. N Engl J Med. 1991;325:1072-1077. 14. Soumerai SB, McLaughlin TJ, Ross-Degnan D, Casteris C, Bollini P. Effects of limiting Medicaid drug-reimbursement benefits on the use of psychotropic agents and acute mental health services by patients with schizophrenia. $N$ Engl J Med. 1994;331:650-655.

15. Epstein AM. Acute myocardial infaretion in the Medicare population: process of care and clinical outcomes. JAMA. 1992;268:2530-2536.

16. Lohr KN. Use of insurance claims data in measuring quality of care. Int $J$ Tech Assess Health Care. 1990;6:263-271.

17. Roos LL, Roos NP, Fisher E, et al. Strengths and Weaknesses of Health Insurance Data Systems for Assessing Ontcomes: Paper Prepared for the Study to Design a Strategy for Quality Review and Assurance in Medicare. Washington, DC: Institute of Medicine, National Academy of Sciences; May 1989. 18. Wennberg JE, Roos NP, Sola L, et al. Use of claims data systems to evaluate health care outcomes: mortality and reoperation following prostatectomy. JAMA. 1987;257:933-936.

19. Sacks H, Chalmers TC, Smith H. Randomized versus historical controls for clinical trials. $A m J$ Med. 1982;72:233-240.

20. Temple R. Problems in the use of large data sets to assess effectiveness. Int J Tech Assess Health Care. 1990;6:211-219.

21. Ray WA, Griffin MR, Baugh DK. Mortality following hip fracture before and after implementation of the prospective payment system. Arch Intern Med. 1990;150:2109-2114.

22. Ray WA, Griffin MR. Use of Medicaid data for pharmacoepidemiology. Am J Epidemiol. 1989;129: 837-849.

23. Soumerai SB, Avorn J, Ross-Degnan D, Gortmaker S. Payment restrictions for prescription drugs under Medicaid: effects on therapy, cost, and equity. $N$ Engl J Med. 1987;317:550-556.

24. Pashos CL, Normand SLT, Garfinkle JB, et al. Trends in the use of drug therapies in patients with acute myocardial infarction: 1988 to $1992 . J \mathrm{Am}$ Coll Cardiol. 1994;23:1023-1030.

25. Fisher ES, Whaley FS, Krushat WM, et al. The accuracy of Medicare's hospital claims data: progress has been made, but problems remain. Am J Public Health. 1992;82:243-248.

26. Iezzoni LI, Burnside S, Sickles L, et al. Coding of acute myocardial infaretion: clinical and policy implications. Ann Intern Med. 1988;109:745-751.

27. Wenneker MB, Epstein AM. Racial inequalities in the use of procedures for patients with ischemic heart disease in Massachusetts. JAMA. 1989; 261:253-257.

28. Ayanian JZ, Epstein AM. Differences in the use of procedures between women and men hospitalized for coronary heart disease. $N E n g l J \mathrm{Med}$. 1991;325:221-225.

29. Wenneker MB, Weissman JS, Epstein AM. The association of payer with utilization of cardiac procedures in Massachusetts. JAMA. 1990;264:12551260.

30. Kalbfleisch JD, Prentice RL. The Statistical Analysis of Failure Time Data. New York, NY: John Wiley \& Sons; 1980.

31. Harrell FE, Pollock BG, Lee KL. Graphical methods for the analysis of survival data. In: Proceedings of the 12 th Annual SAS Users Group International Conference. Cary, NC: SAS Institute Inc; 1988:1107-1115.

32. Harrell FE, Lee KL. Verifying assumptions of the Cox proportional hazards model. In: Proceed ings of the 11th Annual SAS Users Group International Conference. Cary, NC: SAS Institute Inc; 1987:823-828

33. Maldonado G, Greenland S. Simulation study of confounder-selection strategies. Am J Epidemiol. 1993;138:923-936

34. Rosenbaum PR, Rubin DB. Reducing bias in observational studies using subclassification in the pro pensity score. J Am Stat Assoc. 1984;79:516-524.
35. Ayanian J, Hauptman PJ, Guadagnoli E, et al. Knowledge and practices of generalist and specialist physicians regarding drug therapy for acute myocardial infarction. N Engl J Med. 1994;331:11361142.

36. Lamas GA, Pfeffer MA, Hamm P, et al. Do the results of randomized clinical trials of cardiovascular drugs influence medical practice? $N$ Engl $J$ Med. 1992;327:241-247.

37. Kennedy HL, Brooks MM, Barker AM, et al, for the CAST Investigators. Beta-blocker therapy in the Cardiac Arrhythmia Suppression Trial. Am $J$ Cardiol. 1994;74:674-680.

38. McLaughlin TJ, Soumerai SB, Willison DJ, et al. Adherence to national consensus guidelines for drug treatment of suspected acute myocardial infarction in community hospitals: evidence for undertreatment in women and the elderly. Arch Intern Med. 1996;156:799-805.

39. Sial SH, Malone M, Freeman JL, Battiola R, Nachodsky J, Goodwin JS. Beta blocker use in the treatment of community hospital patients discharged after myocardial infarction. J Gen Intern Med. 1994; 9:599-605.

40. Dahlof C, Dimenas E, Kendall M, Wiklund I. Quality of life in cardiovascular diseases: emphasis on $\beta$-blocker treatment. Circulation. 1991;84(suppl VI):VI-108-VI-118.

41. Burris JF. $\beta$-Blockers, dyslipidemia, and coronary artery disease: a reassessment. Arch Intern Med. 1993;153:2085-2092.

42. Ellerbeck EF, Jencks SF, Radford MJ, et al. Quality of care for Medicare patients with acute myocardial infarction: four-state pilot study from the Cooperative Cardiovascular Project. JAMA. 1995;273:1509-1514.

43. F urberg CD, Psaty BM, Meyer JV. Nifedipine: dose-related increase in mortality in patients with coronary heart disease. Circulation. 1995;92:13261331.

44. Horwitz RI, Viscoli CM, Clemens JD, Sadock RT. Developing improved observational methods for evaluating therapeutic effectiveness. Am J Med. 1990;89:630-638. 